\title{
Neutrophil Gelatinase-associated Lipocalin as a Theragnostic Marker in Perihilar Cholangiocarcinoma
}

\author{
AMIT NAIR ${ }^{1}$, NICOLA INGRAM ${ }^{1}$, ELDO T. VERGHESE ${ }^{1}$, IMESHI WIJETUNGA $^{1}$, \\ ALEX F. MARKHAM $^{1}$, JUDY WYATT ${ }^{2}$, K. RAJENDRA PRASAD ${ }^{3}$ and P. LOUISE COLETTA ${ }^{1}$ \\ ${ }^{1}$ Section of Molecular Gastroenterology, Leeds Institute of Medical Research, \\ University of Leeds, Leeds, U.K.; \\ ${ }^{2}$ Department of Histopathology, St. James's University Hospital, Leeds, U.K.; \\ ${ }^{3}$ Department of Hepatobiliary and Transplant Surgery, St. James's University Hospital, Leeds, U.K.
}

\begin{abstract}
Background/Aim: Platforms using valid molecular targets can provide concurrent diagnostic and treatment (theragnostic) options in perihilar cholangiocarcinoma (PHC). Neutrophil gelatinase-associated lipocalin (NGAL) is a biomarker in the biliary secretome of PHC. Its potential as a theragnostic target and its prognostic significance in this cancer was, therefore, explored. Materials and Methods: Invitro studies were used to determine NGAL localization in several cholangiocarcinoma cell lines. Tissue expression of NGAL was quantified in PHC resection cases from 2000-2010 by immunohistochemistry. Results: NGAL was expressed in the majority of tested cell lines and localized to their membranes. Tissues from 54 patients underwent NGAL immunohistochemistry. Median tumoral NGAL expression was significantly higher than that in matched liver controls $(p<0.001)$. Higher NGAL tumor expression was associated with nodal metastasis $(p=0.021)$, although no significant association with survival was observed. Conclusion: The expression and localization of NGAL in PHC make it a valid candidate biomarker for exploitation in theragnostic platforms.
\end{abstract}

Perihilar cholangiocarcinoma (PHC) is a locally aggressive adenocarcinoma of bile duct epithelium arising anatomically at a level between second-order intra-hepatic biliary radicles proximally and the common hepatic duct-cystic duct confluence distally. It is the most common topographical form (up to $50 \%$ ) of cholangiocarcinoma (CCA), whilst the

Correspondence to: Amit Nair, c/o Dr. PL Coletta, Section of Molecular Gastroenterology, Leeds Institute of Medical Research, University of Leeds, Leeds LS9 7TF, U.K. e-mail: P.L.Coletta@leeds.ac.uk

Key Words: Perihilar cholangiocarcinoma, Klatskin tumor, bile duct cancer, neutrophil gelatinase associated lipocalin, molecular targeting, matrix metalloproteinase-9. latter ranks second only to hepatocellular carcinoma as the most common primary hepatic malignancy $(1,2)$. Despite advances in hepatobiliary imaging and chemotherapeutics in the past decade, the poor outcomes of PHC have remained largely unchanged. The characteristic hallmarks of the disease are late diagnosis and poor survival in the absence of complete surgical extirpation (3). Rates of resectability vary widely across the reported literature, ranging from 28 $95 \%$, as do complete resection (R0) rates, which range from $14-95 \%$ (4). Median overall survival (OS) for patients with resected PHC is approximately 38 months (5-year OS=11$42 \%$ ) whereas patients not suitable for resection have a significantly worse median OS of around 12 months. Nodal metastasis, higher tumor stage and positive resection margins are established determinants of poorer OS $(3,4)$.

With a lack of effective adjuvant therapies in PHC, there has been renewed interest in biomarkers of the disease that can be selectively targeted to promote tumor cell death. Interest in particular has been focused on biomarkers in bile, as the latter is considered a 'proximal' fluid in direct contact with PHC tissue which should contain molecules secreted directly into it by tumor cells. However, a biomarker by virtue of its expression in the tumor milieu alone does not render it imminently suitable for concurrent diagnostic and therapeutic (theragnostic) applications. Several key considerations for an ideal theragnostic biomarker exist, most notably its location (tumor cell surface and/or stroma), which facilitates targeting by appropriate systemically administered agents (5).

Following its discovery as a covalently linked complex with the 92-kDa type-IV gelatinase matrix metalloproteinase-9 (MMP9) within human neutrophils, neutrophil gelatinaseassociated lipocalin (NGAL)/lipocalin-2 has emerged as an important molecule in tissue homeostasis. NGAL is a $25-\mathrm{kDa}$ glycoprotein that is a member of a group of secreted transport proteins named lipocalins and is understood to exert antimicrobial effects in vivo, as well as play an important role in 
the regulation of many types of cancer including that of gastric, colorectal and pancreatic origin (6). The impact NGAL has on cancer growth is believed to be mainly via the positive modulatory and stabilizing influence it has on MMP9 extracellularly, although mechanisms independent of MMP9 may also exist such as anti-apoptotic effects on tumor cells (6). NGAL has also recently been identified as a biomarker that is overexpressed in the bile of patients with PHC (7) and more broadly, in those with malignant pancreatobiliary disease (8).

Against this backdrop, this study examined the expression and localization of NGAL in a panel of CCA cell lines using western blotting and enzyme-linked immunosorbent assay (ELISA) techniques to assess the preliminary validity and suitability of this biomarker as a candidate for tumor targeting in PHC. In addition, immunohistochemistry (IHC) was utilized to determine the impact of expression of NGAL and MMP9 on clinicopathological aspects of PHC.

\section{Materials and Methods}

Cell lines. The PHC cell line OZ (JCRB, Osaka, Japan) (9), and extrahepatic CCA cell lines EGI-1, TFK-1 (DMSZ, Braunschweig, Germany) (10), and SkChA-1 (11) were sourced and maintained by serial cell culture. For comparative purposes, intra-hepatic CCA cell lines Huh-28 and HuCCT-1 (JCRB) $(12,13)$ and the normal cholangiocyte cell line H69 (14) were similarly maintained and also subjected to the same assays. All cell lines were authenticated inhouse using short tandem repeat analysis.

Western blotting. Whole-cell lysates were made from confluent cultures of all seven cell-lines. Lysates enriched for cell membrane proteins were similarly collected using the ProteoExtract ${ }^{\circledR}$ Transmembrane protein extraction kit (Novagen, San Diego, CA, USA) as per manufacturer's instructions. Cytoplasmic extracts were obtained as a by-product of this process. Lysates were electrophoretically separated in $12 \%$ reducing SDS-PAGE (Novex, San Diego, CA, USA). Following protein transfer to membrane, the anti-NGAL goat polyclonal antibody (R\&D Systems, Minneapolis, MN, USA) diluted to $0.1 \mu \mathrm{g} / \mathrm{ml}$ was applied for 1 hour at room temperature. For assessment of equal protein loading, the mouse monoclonal antibody $\beta$-actin $(1 / 20,000$; Sigma-Aldrich, St Louis, MO, USA) or mouse monoclonal $\alpha$-tubulin (1/5000; Sigma-Aldrich) was employed. After washing, the appropriate secondary antibody was applied at $1 / 2000$ dilution.

Sandwich ELISA for NGAL quantitation in cell culture supernatants. The Quantikine NGAL ELISA kit (R\&D Systems, Minneapolis, MN, USA) was employed as per the manufacturer's directions for quantifying NGAL secretion into tested cell culture supernatants.

Immunohistochemistry and patient database. Ethical approval (REC 06/Q1206/136) was obtained from the Leeds (East) National Research Ethics Service Committee for the study of archival PHC tissue samples. A list of consecutive patients who underwent resection of PHC at our Institution from January 2000 to September 2010 was collated from a retrospective database and used to source formalin-fixed Paraffin embedded tissue blocks. Where available, for each case a block consisting of adjacent normal liver tissue was also procured for control staining purposes. Clinicopathological parameters were collected from patient records. Tumors were staged again as per the seventh edition of the American Joint Committee on Cancer guidelines on perihilar bile ducts (15). The time to tumor recurrence and date of last follow-up or death was noted for each patient. Analysis was conducted as per REMARK guidelines (16).

Five-micron-thick tissue sections were dewaxed and hydrated. No antigen retrieval was required. Endogenous peroxidases were quenched with $0.3 \% \mathrm{v} / \mathrm{v}$ hydrogen peroxide. Prior to antibody application, sections for NGAL staining were subject to blocking with Avidin-Biotin, followed by 1:10 Casein solution (both Vector, Burlingame, CA, USA). Sequential washes with tris-buffered saline, and tris-buffered saline-Tween were used between all steps. The primary antibodies utilized were goat polyclonal anti-NGAL (1/100; R\&D Systems) and mouse monoclonal anti-MMP9 (1/100; Abcam, Cambridge, UK), each of which was applied for 1 hour at room temperature. NGAL sections were further subjected to secondary incubation with a rabbit anti-goat biotinylated immunoglobulin $(1 / 200$; Dako, Carpinteria, CA, USA) for 30 minutes. Vectastain ${ }^{\circledR}$ Elite ${ }^{\circledR}$ ABC kit (Vector) was then added to these sections. In the case of MMP9, Dako EnVision $^{\mathrm{TM}}$ System-HRP for use with Mouse primary antibodies (Dako) was utilized for secondary incubation for $30 \mathrm{~min}$. Finally, all sections were treated with 3,3'-diaminobenzidine, hematoxylin and serial dehydration prior to mounting.

Histopathological analysis. For quantitation of MMP9 and NGAL IHC staining, the Histoscore method (17) was adopted. Briefly, PHC tumor tissue was first identified in each section at $\times 40$ magnification. Subsequently at $\times 400$ magnification $(1$ high-power field), a maximum of 100 tumor epithelial cells were assessed for their intensity of staining, ranging from 0 (none) to 3 (highest) per cell. This step was repeated across 10 high-power fields and the total number of cells for each intensity was recorded. The Histoscore was calculated using the formula: $H=P(\mathrm{I}+1)$, where $H$ is the histoscore for a given intensity, $P$ was the percentage of positiveIy stained cells for a given intensity and I was the intensity.

The net sum of Histoscores for all intensities per section yielded the final Histoscore (ranging from 100-400), which was recorded. The process was similarly performed for matched control liver tissue. In the latter instance, control hepatocytes were assessed rather than bile ducts, since off-target effects of any theragnostic platform to the liver should predominantly affect the most numerous cells in the vicinity of tumor (provided they too express the candidate biomarker) viz. normal surrounding hepatocytes. Scores were independently calculated by two observers (AN, ETV) for the first 20 cases to ensure adequate interobserver correlation, after which the remainder of cases were assessed by a single scorer (AN). Both observers were blinded to clinical data and matched tissue at the time of scoring any particular slide.

Statistical analysis. The statistical software package SPSS v22 (IBM, Armonk, NY, USA) was used for data analysis, with a $p$-value of less than 0.05 used to denote statistical significance. Non-parametric tests were employed for analysis of appropriate factors. Correlations between continuous variables were assessed with the Spearman test, and the Wilcoxon signed-rank test was utilized for matched tissue comparisons. Using an online tool (http:// molpath.charite.de/cutoff) (18), analysis of the area under the curve (AUC) of the receiver operating characteristics (ROC) of NGAL Histoscores was performed to determine optimal cut-off points predictive of appropriate endpoints. 
A

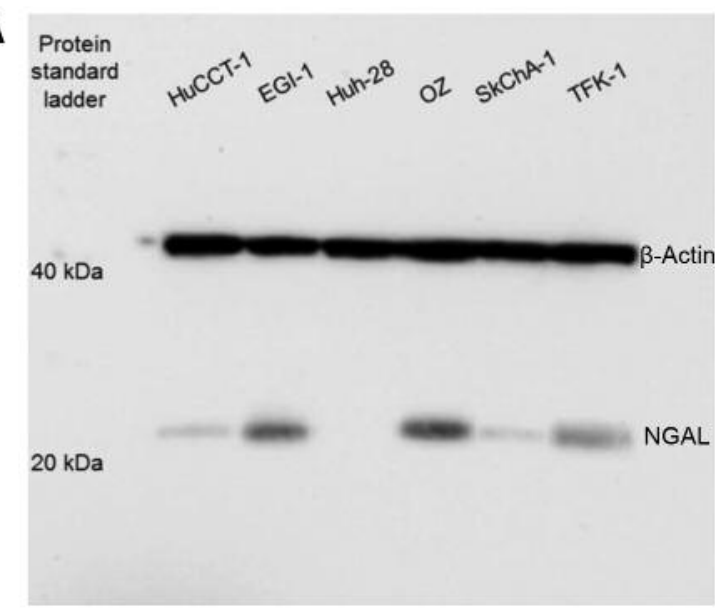

B

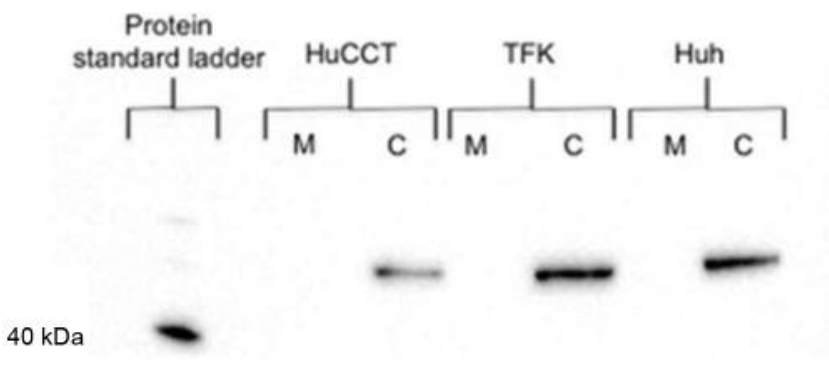

C
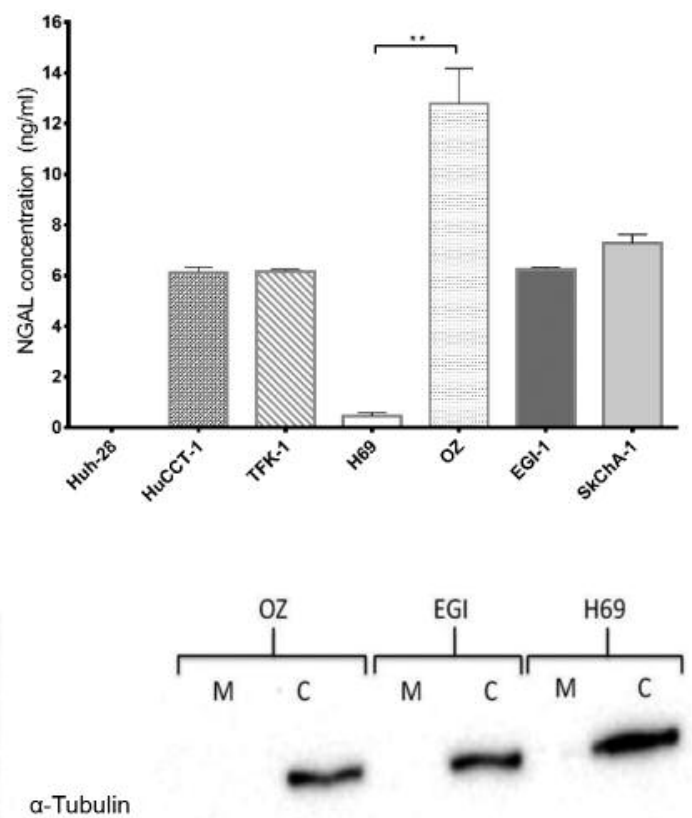

$20 \mathrm{kDa}$

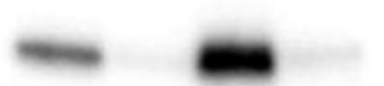

NGAL

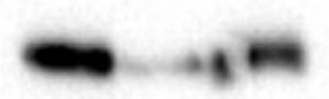

Figure 1. Comparison of in vivo expression profile of neutrophil gelatinase-associated lipocalin (NGAL) in tested cholangiocarcinoma (CCA) cell lines. A: Western blot image demonstrating expression of NGAL protein $(25 \mathrm{kDa})$ in whole-cell lysates of cell lines. The absence of expression by the intrahepatic CCA cell line Huh-28 is evident, whereas OZ showed the strongest expression. $\beta$-Actin (42 kDa) was used as a loading control. B: Western blot image highlighting NGAL protein in membrane-enriched lysates of CCA and H69 cell lines. Preferential expression of NGAL (25kDa) in the membrane portion (' $M$ ') of fractionated cell lysates is seen in comparison to their respective cytoplasmic equivalents (' $C$ '). Expression is notably absent from Huh-28 and the normal cholangiocyte cell line H69. $\alpha$-Tubulin $(50 \mathrm{kDa})$ was used as loading control. C: Enzyme-linked immunosorbent assay results showing NGAL concentrations in CCA and H69 cell culture supernatants. Minimal secretion was seen in H69, whereas Huh-28 showed no expression (the latter was therefore omitted from statistical analysis). Pooled data from three runs are shown; data are median values and error bars indicate inter-quartile range. ${ }^{* *} p=0.009$ on pairwise comparison (Kruskal-Wallis test).

This tool was also utilized for dichotomizing Histoscores for survival analysis. Survival curves were generated using the Kaplan-Meier method and differences assessed using the log-rank test. Censoring of survival data was performed for all cases of peri-operative death, patients who died due to unrelated causes or were lost to follow-up and in those alive at the end of 5 years of follow-up.

\section{Results}

NGAL exhibits membranous expression and extracellular secretion in cholangiocarcinoma. Western blotting analysis of whole-cell lysates revealed the presence of NGAL in all tested CCA cell lines at the expected molecular weight of $25 \mathrm{kDa}$, with the exception of the intra-hepatic cell line Huh-28 (Figure
1A). Fractionated lysates showed predominant expression of NGAL in the membrane fraction of CCA cell lines EGI-1, TFK-1 and OZ (Figure 1B). Notably, the normal cholangiocyte cell line H69 did not express any appreciable level of NGAL.

Results of ELISA testing mirrored those seen by western blotting, with PHC and extra-hepatic CCA cell line supernatants showing higher concentrations of NGAL, in comparison to Huh-28 and H69 (Figure 1C).

NGAL and MMP9 are significantly overexpressed in PHC in comparison to control liver tissue. A total of 54 patients underwent resection of PHC during the study period. Patient clinicopathological variables are summarized in Table I. 
Median and inter-quartile range (IQR) of NGAL and MMP9 tumor Histoscores across all cases were 327 (282-366) and 284 (243-323) respectively. All tumor samples showed expression of both markers to varying degrees. The staining pattern observed in tumor parenchyma showed NGAL and MMP9 to be localized to both cell membrane and to the basal aspect of the cytoplasm, along with variable degrees of expression in peri-tumoral stroma (Figure 2A).

NGAL expression is associated with nodal metastasis and local recurrence. Histoscore analysis of sections showed an appreciably higher level of NGAL expression in tumor tissue when compared to matched control liver tissue (hepatocytes), whereas for MMP9, this difference only just achieved significance (Figure 2B). No significant correlation was found between the degree of NGAL and MMP9 tumoral expression in this cohort (Figure 2C, left). There were no instances of negative staining of liver controls for either NGAL or MMP9. Interestingly, lower median NGAL and MMP9 Histoscores were significantly associated with local recurrence (defined as recurrent disease within the surgical bed). No other patient variables bore any significant association with tumor Histoscores of either biomarker. In the analysis of control liver tissue, no correlation was found between pre-operative serum bilirubin levels and the NGAL Histoscores (Figure 2C, right).

Table II demonstrates the relations between tissue expression of NGAL and MMP9 and clinicopathological data. The median NGAL Histoscore was significantly higher in patients with nodal metastasis. AUC-ROC analysis showed that an NGAL Histoscore cut-off of $>345$ was best at identifying patients with lymph nodal metastasis, with sensitivity and specificity of 59\% and $80 \%$ respectively. No prognostic impact of NGAL or MMP9 was evident in this cohort

The median OS for the entire cohort overall was 23.5 months. Utilizing the NGAL Histoscore cut-off of 345 (associated with nodal metastasis), no prognostic impact on OS (Figure 3, left) or disease-free survival was evident $(p=0.37$; data not shown). Neither NGAL nor MMP9 Histoscores achieved any cut-off value indicative of a prognostic impact on OS or disease-free survival. In addition, the occurrence of nodal metastasis notably also did not have an impact on survival in the study group (Figure 3, right).

\section{Discussion}

The exact roles played by NGAL in the progression of cancer are yet to be fully elucidated. Data regarding the prognostic impact of NGAL expression in solid cancer is disparate at best, with both pro-and anticancer effects noted in cancer of the breast, esophagus, stomach, and colon (6, $19)$, as well as hepatocellular carcinoma $(20,21)$. The impetus towards examining the characteristics of NGAL in
Table I. Summary of clinicopathological patient variables (total=54).

\begin{tabular}{|c|c|}
\hline Variable & Value \\
\hline \multicolumn{2}{|l|}{ Age, years } \\
\hline Median (range) & $57.6(33-81)$ \\
\hline \multicolumn{2}{|l|}{ Gender } \\
\hline Male $(\%)$ & $31(57.4)$ \\
\hline Female $(\%)$ & $23(42.6)$ \\
\hline \multicolumn{2}{|l|}{ Surgical procedure, $\mathrm{n}(\%)$} \\
\hline Left extended hepatectomy $(\mathrm{S} 2,3,4,5,8, \pm 1)+\mathrm{BDE}$ & $18(33)$ \\
\hline Left hemihepatectomy $(\mathrm{S} 2,3,4, \pm 1)+\mathrm{BDE}$ & $7(13)$ \\
\hline Right extended hepatectomy (S4, 5, 6, 7, 8, \pm 1$)+\mathrm{BDE}$ & $19(35)$ \\
\hline Right hemihepatectomy $(\mathrm{S} 5,6,7,8, \pm 1)+\mathrm{BDE}$ & $7(13)$ \\
\hline Orthotopic liver transplant & $2(4)$ \\
\hline BDE alone & $1(2)$ \\
\hline \multicolumn{2}{|l|}{ Tumor size, mm } \\
\hline Median (range) & $30(10-75)$ \\
\hline \multicolumn{2}{|l|}{ Tumor differentiation (grade), $\mathrm{n}(\%)$} \\
\hline Well (G1) & $27(50)$ \\
\hline Moderate (G2) & $19(35.2)$ \\
\hline Poor (G3) & $8(14.8)$ \\
\hline \multicolumn{2}{|l|}{ Vascular invasion, n (\%) } \\
\hline Microscopic & $33(61.1)$ \\
\hline \multicolumn{2}{|l|}{ Perineural infiltration, $\mathrm{n}(\%)$} \\
\hline Perineural infiltration & $48(88.9)$ \\
\hline \multicolumn{2}{|l|}{ Tumor stage, n (\%) } \\
\hline $\mathrm{T} 1$ & $2(3.7)$ \\
\hline $\mathrm{T} 2$ & $29(53.7)$ \\
\hline $\mathrm{T} 3$ & $19(35.2)$ \\
\hline $\mathrm{T} 4$ & $4(7.4)$ \\
\hline \multicolumn{2}{|l|}{ Nodal stage, n (\%) } \\
\hline No & $25(46.3)$ \\
\hline N1 & $21(38.9)$ \\
\hline $\mathrm{N} 2$ & $8(14.8)$ \\
\hline \multicolumn{2}{|l|}{ AJCC Stage (7th edition), n (\%) } \\
\hline I & $0(0)$ \\
\hline II & $15(27.8)$ \\
\hline IIIA & $8(14.8)$ \\
\hline IIIB & $21(38.9)$ \\
\hline IVA & $2(3.7)$ \\
\hline IVB & $8(14.8)$ \\
\hline \multicolumn{2}{|l|}{ Resection margin, $\mathrm{n}(\%)$} \\
\hline R0 (clear) & $21(38.9)$ \\
\hline R1 (involved) & $33(61.1)$ \\
\hline \multicolumn{2}{|l|}{ Local recurrence, n (\%) } \\
\hline In surgical bed & $5(9.3)$ \\
\hline
\end{tabular}

AJCC: American Joint Committee on Cancer; BDE: bile duct excision; S: segment.

PHC in this study stemmed from prior biliary proteomics work at our Institution showing NGAL to be increased in bile of patients with PHC (7). We have shown NGAL is expressed more by PHC and extra-hepatic CCA cell lines than in normal cholangiocytes, and that expression is predominantly in the membrane fraction of these cells. Evidence of extracellular secretion was also observed. These findings were mirrored on IHC analysis of PHC tissue, where we found NGAL to be significantly overexpressed, 
A
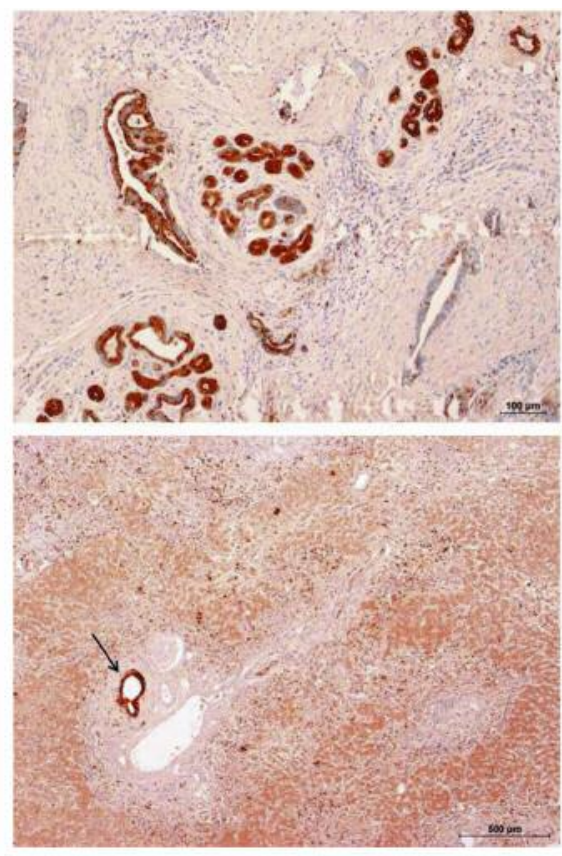

B

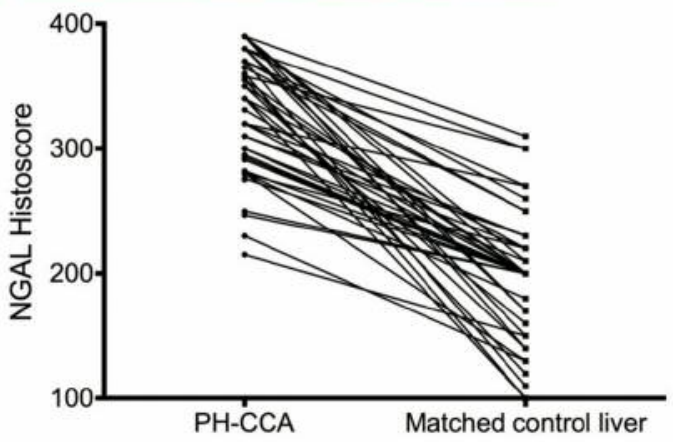

C

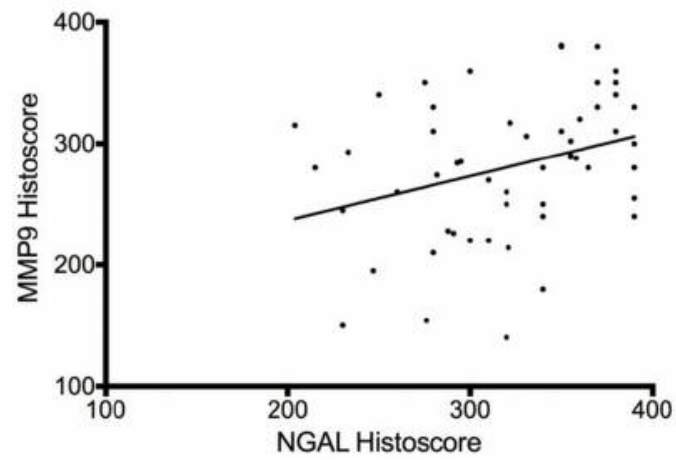

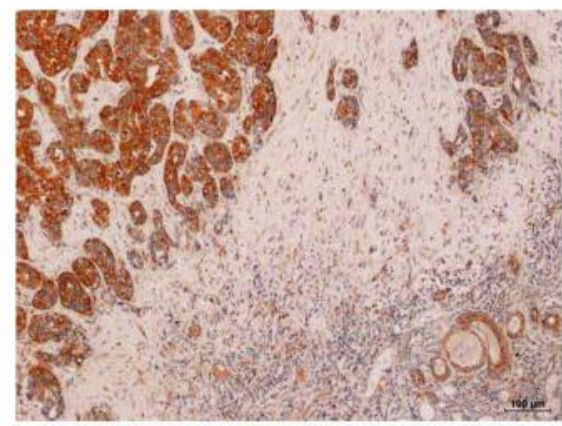
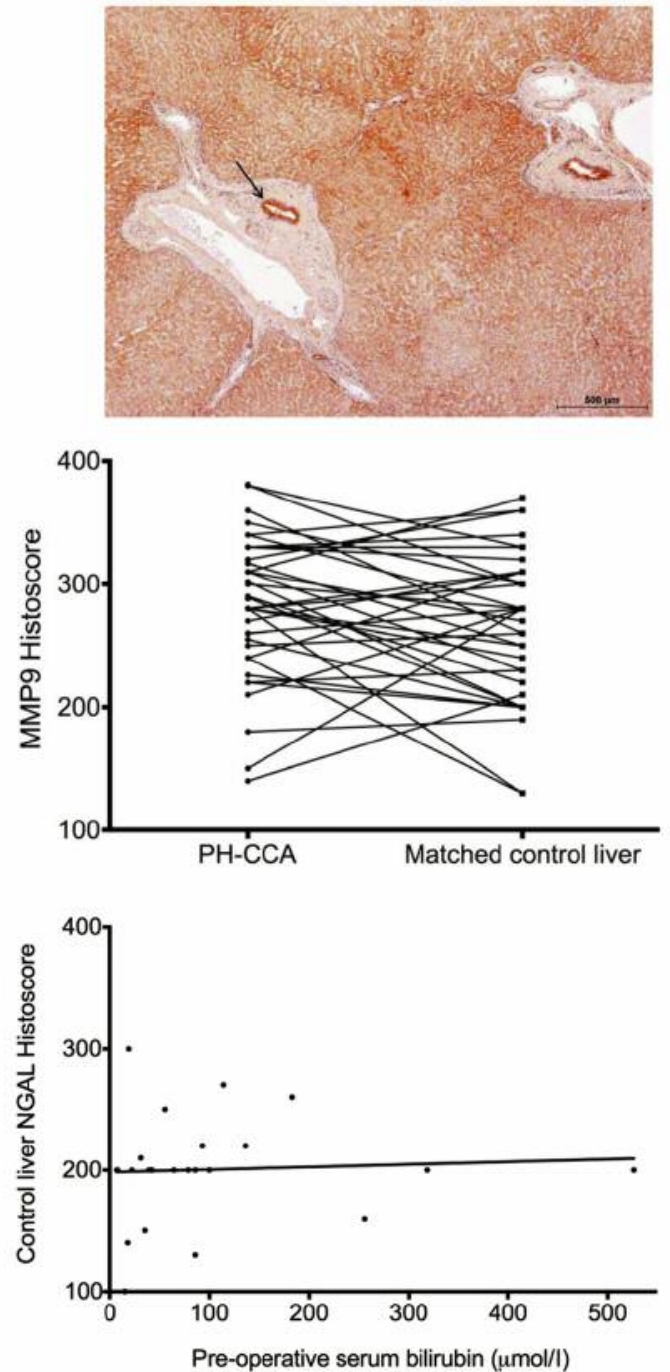

Figure 2. Tissue expression profiles of neutrophil gelatinase-associated lipocalin (NGAL) and matrix metalloproteinase 9 (MMP9) in cholangiocarcinoma. A: Left: Strong NGAL expression was observed in perihilar cholangiocarcinoma (PHC) glandular tissue with mild positivity in tumor stroma (upper panel; scale bar=100 $\mu \mathrm{m}$ ). Diffuse NGAL staining of matched normal liver parenchyma (hepatocytes) was found (lower panel; scale bar $=500 \mu \mathrm{m}$ ), with strong positivity of biliary radicles (arrow) within a portal triad. Right: Similarly, strong expression of MMP9 in PHC glandular tissue was seen with mild stromal staining (upper panel). A substantial degree of parenchymal expression is apparent in matched normal liver tissue (hepatocytes) (lower panel), and within normal cholangiocytes (arrow). B: Histoscores for NGAL (left) and MMP9 (right) in tumor versus matched control liver tissue. NGAL Histoscore in tumor tissue was significantly higher compared to control liver $(n=44 ; p<0.001$, Wilcoxon signedrank test). Tumor tissue expression of MMP9 was higher than in controls, but only just achieving statistical significance $(n=39 ; p=0.049 ;$ Wilcoxon signed-rank test). C: Left: NGAL versus MMP9 Histoscores in PHC tissue, with the line of best fit shown. The correlation of Histoscores between these two markers was low (Spearman $r=0.3 ; p=0.017$ ). Right: NGAL Histoscores plotted against pre-operative serum bilirubin, with the line of best fit shown. Poor agreement between these variables is observed, this finding was not statistically significant ( $n=23$; Spearman $r=0.22 ; p=0.31$ ). 

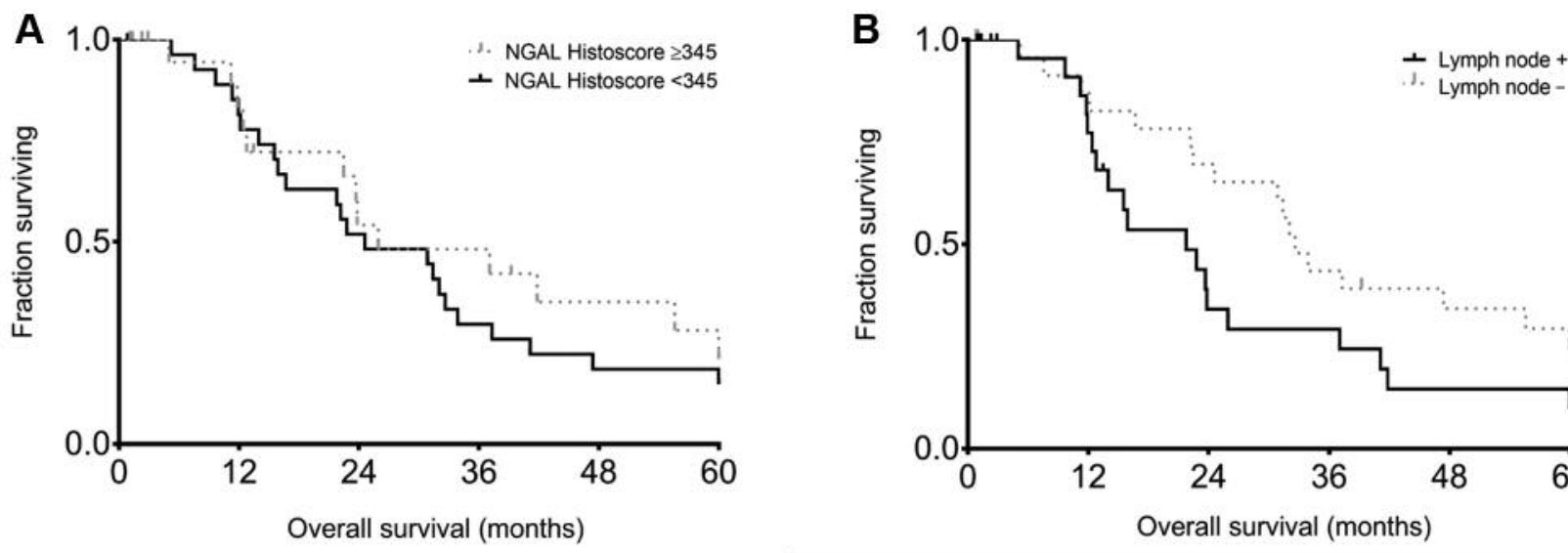

\begin{tabular}{lllllll}
\hline NGAL $\geq 345$ & 22 & 15 & 9 & 8 & 5 & 4 \\
\hline NGAL $<345$ & 32 & 22 & 14 & 8 & 5 & 5
\end{tabular}

\begin{tabular}{ccccccc}
\multicolumn{7}{c}{ Overall survival (months) } \\
\hline Lymph node + & 29 & 17 & 7 & 6 & 3 & 3 \\
\hline Lymph node - & 25 & 20 & 16 & 10 & 7 & 6 \\
\hline
\end{tabular}

Figure 3. A: Kaplan-Meier curve for overall survival grouped by neutrophil gelatinase-associated lipocalin (NGAL) Histoscore cut-off of 345. No significant difference was seen in this instance ( $p=0.42$; log-rank test). B: Kaplan-Meier curve for overall survival based on nodal metastasis. No significant difference was observed between node-positive and-negative patient groups ( $p=0.07 ;$ log-rank test). The number of patients at risk at corresponding time points is shown in the table below each graph.

Table II. Comparison of neutrophil gelatinase-associated lipocalin (NGAL) and matrix metalloproteinase 9 (MMP9) Histoscores by clinicopathological patient variables.

\begin{tabular}{|c|c|c|c|c|c|c|}
\hline Variable & Variable & $\mathrm{n}(\%)$ & $\begin{array}{l}\text { NGAL Histoscore } \\
\text { [median (IQR)] }\end{array}$ & $p$-Value* & $\begin{array}{l}\text { MMP9 Histoscore } \\
\text { [median (IQR)] }\end{array}$ & $p$-Value* \\
\hline \multirow[t]{2}{*}{ Age } & $<58$ Years & $27(50)$ & $321(280-370)$ & \multirow[t]{2}{*}{0.78} & $285(250-330)$ & \multirow[t]{2}{*}{0.67} \\
\hline & $\geq 58$ Years & $27(50)$ & $340(288-365)$ & & $280(240-320)$ & \\
\hline \multirow[t]{2}{*}{ Gender } & Male & $31(57)$ & $310(280-360)$ & \multirow[t]{2}{*}{0.21} & $280(228-317)$ & \multirow[t]{2}{*}{0.23} \\
\hline & Female & $23(43)$ & $340(295-370)$ & & $293(250-340)$ & \\
\hline \multirow[t]{2}{*}{ Tumor size (mm) } & $<30 \mathrm{~mm}$ & $24(46)$ & $321(284-357)$ & \multirow[t]{2}{*}{0.97} & $280(220-317)$ & \multirow[t]{2}{*}{0.35} \\
\hline & $\geq 30 \mathrm{~mm}$ & $28(54)$ & $340(276-369)$ & & $282(253-328)$ & \\
\hline \multirow[t]{2}{*}{ Histological grade } & 1 & $27(50)$ & $322(280-370)$ & \multirow[t]{2}{*}{0.75} & $300(226-330)$ & \multirow[t]{2}{*}{0.74} \\
\hline & $2 / 3$ & $27(50)$ & $331(282-358)$ & & $284(255-306)$ & \\
\hline \multirow[t]{2}{*}{ Microscopic vascular invasion } & Yes & $33(65)$ & $322(280-375)$ & \multirow[t]{2}{*}{0.79} & $289(242-335)$ & \multirow[t]{2}{*}{0.34} \\
\hline & No & $18(35)$ & $336(289-366)$ & & $280(224-311)$ & \\
\hline \multirow[t]{2}{*}{ Nodal metastases } & Yes & $29(54)$ & $355(296-380)$ & \multirow[t]{2}{*}{0.02} & $290(252-330)$ & \multirow[t]{2}{*}{0.35} \\
\hline & No & $25(46)$ & $310(278-340)$ & & $280(240-316)$ & \\
\hline \multirow[t]{2}{*}{ T Stage (AJCC 7th ed) } & $\mathrm{T} 1 / \mathrm{T} 2$ & $31(57)$ & $322(276-355)$ & \multirow[t]{2}{*}{0.21} & $285(240-317)$ & \multirow[t]{2}{*}{0.77} \\
\hline & $\mathrm{T} 3 / \mathrm{T} 4$ & $23(43)$ & $340(250-380)$ & & $284(245-330)$ & \\
\hline \multirow[t]{2}{*}{ Resection margins } & R0 (positive) & $21(39)$ & $340(286-380)$ & \multirow[t]{2}{*}{0.53} & $280(233-324)$ & \multirow[t]{2}{*}{0.58} \\
\hline & R1 (negative) & $33(61)$ & $321(281-362)$ & & $289(247-325)$ & \\
\hline \multirow[t]{2}{*}{ Local recurrence } & Yes & $5(9)$ & $280(253-304)$ & \multirow[t]{2}{*}{0.03} & $228(182-248)$ & \multirow[t]{2}{*}{0.005} \\
\hline & No & $49(91)$ & $340(292-370)$ & & $289(258-330)$ & \\
\hline \multirow[t]{2}{*}{ Metastasis } & Yes & $26(48)$ & $340(292-378)$ & \multirow[t]{2}{*}{0.21} & $292(256-323)$ & \multirow[t]{2}{*}{0.32} \\
\hline & No & $28(52)$ & $321(272-359)$ & & $280(230-325)$ & \\
\hline
\end{tabular}

AJCC: American Joint Committee on Cancer; IQR: interquartile range. *Mann-Whitney $U$-test.

along with tumor stromal positivity. Clinicopathological variables significantly associated with NGAL expression were nodal metastasis and local recurrence, although on survival analysis, NGAL had no significant impact. As far as we are aware of, our report is the first to examine both the cellular localization of this marker in PHC and its prognostic bearing. Under the broader umbrella of CCA, an earlier report examined NGAL expression in a cohort of 24 cases 
and noted no association between tissue expression and variables such as tumor differentiation, lymph nodal status or metastases (22). In a more recent study, increased NGAL tissue expression was significantly associated with positive resection margins (but not with tumor size, differentiation or nodal status) and found to confer a poorer prognosis within a cohort of 78 patients with CCA (23).

Intracellular iron depletion leading to apoptosis is understood to be one of the mechanisms by which NGAL induces cancer cell death. NGAL also functions as a MMP9 stabilizer in the extracellular matrix which helps promote tumor invasiveness in certain cancer types by virtue of MMP9 gelatinase activity (6). Paradoxically, NGAL also exerts anticancer effects in some types of cancer through the inhibition of vascular endothelial growth factor synthesis and by the inhibition of focal adhesion kinase phosphorylation (19). The close interplay between NGAL and MMP9 prompted us also to analyze expression of the latter by IHC, where we found it too was associated with local recurrence, but not survival. No significant correlation between NGAL and MMP9 expression was found. The available data on MMP9 expression in PHC is scant, with existing studies noting an association between overexpression and neural invasion (24), poorer survival (25) and even improved prognosis (26).

The reasons for NGAL overexpression being linked with nodal metastasis, with lower expression being associated with local recurrence are not entirely clear. This may be indicative of mutually exclusive roles of the molecule in these processes. Additionally, although NGAL was significantly linked with nodal metastasis, the sensitivity of a NGAL Histoscore cut-off of 345 for this event was only 59\%. It is also noteworthy that we were also unable to demonstrate poorer survival in those with nodal metastasis [an established prognostic factor in this cancer in larger series $(27,28)]$. Both these observations are perhaps best explained by the modest size of our study group despite 10 years' worth of case accrual in a Western high resection volume center. One can only surmise that NGAL may indeed have a prognostic impact in PHC when analyzed in larger data sets.

Given the varied role of NGAL in homoeostasis, it is not surprising that its expression was noted in normal liver tissue. Previous studies have nonetheless noted a lack of NGAL staining in normal hepatocytes $(22,29)$. However, under conditions of experimental liver injury or cholestasis, tissue and serum NGAL expression levels were observed to be markedly increased, sourced from injured hepatocytes (30). As $\mathrm{PHC}$ is classically associated with jaundice at presentation, we therefore assessed correlation between pre-operative serum bilirubin and NGAL Histoscore in control liver, but were unable to demonstrate any significant association.

Theragnostic biomarkers should ideally fulfil certain criteria that improve the chances of successful molecular targeting in cancer. Such factors include hyper-expression in target tumor tissue, membranous/extracellular localization and increased prevalence of the marker in the affected population (5). We demonstrated here that NGAL fulfils these characteristics. Molecular imaging of animal models of pancreatic cancer utilizing an anti-NGAL strategy has already been attempted with success, although low signal specificity and positivity in other tissues were drawbacks $(31,32)$.

In summary, our results indicate that NGAL is a valid theragnostic target in PHC, although off-target effects can be encountered with a pure anti-NGAL approach. One possible way to lessen the impact of lateral effects is to confer it or any candidate theragnostic agent with further tumor specificity using an additional layer of molecular targeting against tumor vascular endothelium $(33,34)$. The results of such a hybrid approach will hopefully provide new inroads to the treatment of this aggressive cancer.

\section{Acknowledgements}

This work was supported by a grant from the Rays of Hope charity, Leeds Teaching Hospitals Charitable Foundation, UK.

\section{References}

1 Khan SA, Davidson BR, Goldin RD, Heaton N, Karani J, Pereira SP, Rosenberg WM, Tait P, Taylor-Robinson SD, Thillainayagam AV, Thomas $\mathrm{HC}$ and Wasan $\mathrm{H}$ : Guidelines for the diagnosis and treatment of cholangiocarcinoma: An update. Gut 61(12): 1657-1669, 2012.

2 Razumilava N and Gores GJ: Cholangiocarcinoma. Lancet 383(9935): 2168-2179, 2014.

3 Groot Koerkamp B and Fong Y: Outcomes in biliary malignancy. J Surg Oncol 110(5): 585-591, 2014.

4 Zhang W and Yan LN: Perihilar cholangiocarcinoma: Current therapy. World J Gastrointest Pathophysiol 5(3): 344-354, 2014.

5 van Oosten M, Crane LM, Bart J, van Leeuwen FW and van Dam GM: Selecting potential targetable biomarkers for imaging purposes in colorectal cancer using target selection criteria (TASC): A novel target identification tool. Transl Oncol 4(2): 71-82, 2011.

6 Chakraborty S, Kaur S, Guha S and Batra SK: The multifaceted roles of neutrophil gelatinase-associated lipocalin (NGAL) in inflammation and cancer. Biochim Biophys Acta 1826(1): 129$169,2012$.

7 Farid SG, Craven RA, Peng J, Bonney GK, Perkins DN, Selby PJ, Rajendra Prasad K and Banks RE: Shotgun proteomics of human bile in hilar cholangiocarcinoma. Proteomics 11(10): 2134-2138, 2011

8 Zabron AA, Horneffer-van der Sluis VM, Wadsworth CA, Laird F, Gierula M, Thillainayagam AV, Vlavianos P, Westaby D, Taylor-Robinson SD, Edwards RJ and Khan SA: Elevated levels of neutrophil gelatinase-associated lipocalin in bile from patients with malignant pancreatobiliary disease. Am J Gastroenterol 106(9): 1711-1717, 2011.

9 Homma S, Nagamori S, Fujise K, Yamazaki K, Hasumura S, Sujino H, Matsuura T, Shimizu K, Kameda H and Takaki K: Human bile duct carcinoma cell line producing abundant mucin in vitro. Gastroenterologia Japonica 22(4): 474-479, 1987. 
10 Saijyo S, Kudo T, Suzuki M, Katayose Y, Shinoda M, Muto T, Fukuhara K, Suzuki T and Matsuno S: Establishment of a new extrahepatic bile duct carcinoma cell line, TFK-1. Tohoku J Exp Med 177(1): 61-71, 1995.

11 Knuth A, Gabbert H, Dippold W, Klein O, Sachsse W, BitterSuermann D, Prellwitz W and Meyer zum Buschenfelde KH: Biliary adenocarcinoma. Characterisation of three new human tumor cell lines. J Hepatol 1(6): 579-596, 1985.

12 Kusaka Y, Muraoka A, Tokiwa T and Sato J: Establishment and characterization of a human cholangiocellular carcinoma cell line. Human Cell 1(1): 92-94, 1988 (in Japanese).

13 Miyagiwa M, Ichida T, Tokiwa T, Sato J and Sasaki H: A new human cholangiocellular carcinoma cell line (HUCC-T1) producing carbohydrate antigen 19/9 in serum-free medium. In Vitro Cell Develop Biol 25(6): 503-510, 1989.

14 Grubman SA, Perrone RD, Lee DW, Murray SL, Rogers LC, Wolkoff LI, Mulberg AE, Cherington V and Jefferson DM: Regulation of intracellular $\mathrm{pH}$ by immortalized human intrahepatic biliary epithelial cell lines. Am J Physiol 266(6 Pt 1): G1060-1070, 1994.

15 Edge SB, American Joint Committee on Cancer. and American Cancer Society: AJCC Cancer Staging Handbook: From the AJCC Cancer Staging Manual. 7th edn. Springer: New York, 2010.

16 Altman DG, McShane LM, Sauerbrei W and Taube SE: Reporting recommendations for tumor marker prognostic studies (REMARK): Explanation and elaboration. PLoS Med 9(5): e1001216, 2012.

17 Jonat W, Maass $\mathrm{H}$ and Stegner HE: Immunohistochemical measurement of estrogen receptors in breast cancer tissue samples. Cancer Res 46(8 Suppl): 4296s-4298s, 1986.

18 Budczies J, Klauschen F, Sinn BV, Gyorffy B, Schmitt WD, Darb-Esfahani S and Denkert C: Cutoff finder: A comprehensive and straightforward web application enabling rapid biomarker cutoff optimization. PLoS One 7(12): e51862, 2012.

19 Candido S, Abrams SL, Steelman LS, Lertpiriyapong K, Fitzgerald TL, Martelli AM, Cocco L, Montalto G, Cervello M, Polesel J, Libra M and McCubrey JA: Roles of NGAL and MMP-9 In the tumor microenvironment and sensitivity to targeted therapy. Biochim Biophys Acta 1863(3): 438-448, 2016.

20 Wang YP, Yu GR, Lee MJ, Lee SY, Chu IS, Leem SH and Kim DG: Lipocalin-2 negatively modulates the epithelial-tomesenchymal transition in hepatocellular carcinoma through the epidermal growth factor (TGF-beta1)/LCN2/TWIST1 pathway. Hepatology 58(4): 1349-1361, 2013.

21 Zhang Y, Fan Y and Mei Z: NGAL and NGALR overexpression in human hepatocellular carcinoma toward a molecular prognostic classification. Cancer Epidemiol 36(5): e294-299, 2012.

22 Nuntagowat C, Leelawat K and Tohtong R: Ngal knockdown by siRNA in human cholangiocarcinoma cells suppressed invasion by reducing NGAL/MMP-9 complex formation. Clin Exp Metast 27(5): 295-305, 2010.

23 Chiang KC, Yeh TS, Wu RC, Pang JS, Cheng CT, Wang SY, Juang $\mathrm{HH}$ and Yeh CN: Lipocalin 2 (LCN2) is a promising target for cholangiocarcinoma treatment and bile LCN2 level is a potential cholangiocarcinoma diagnostic marker. Sci Rep 6: 36138, 2016.
24 Kirimlioglu H, Turkmen I, Bassullu N, Dirican A, Karadag N and Kirimlioglu V: The expression of matrix metalloproteinases in intrahepatic cholangiocarcinoma, hilar (Klatskin tumor), middle and distal extrahepatic cholangiocarcinoma, gallbladder cancer, and ampullary carcinoma: Role of matrix metalloproteinases in tumor progression and prognosis. Turk J Gastroenterol 20(1): 4147, 2009.

25 Sun Q, Zhao C, Xia L, He Z, Lu Z, Liu C, Jia M, Wang J and Niu J: High expression of matrix metalloproteinase-9 indicates poor prognosis in human hilar cholangiocarcinoma. Int J Clin Exp Pathol 7(9): 6157-6164, 2014.

26 Park Y, Kim K, Paik JH, Chie EK, Jang JY, Kim SW and Oh DY: High expression of MMP-9 is associated with better prognosis in extrahepatic bile duct cancer patients. Eur J Surg Oncol 44(5): 638-643, 2018.

27 DeOliveira ML, Cunningham SC, Cameron JL, Kamangar F, Winter JM, Lillemoe KD, Choti MA, Yeo CJ and Schulick RD: Cholangiocarcinoma: Thirty-one-year experience with 564 patients at a single institution. Ann Surg 245(5): 755-762, 2007.

28 Nagino M, Ebata T, Yokoyama Y, Igami T, Sugawara G, Takahashi Y and Nimura Y: Evolution of surgical treatment for perihilar cholangiocarcinoma: A single-center 34-year review of 574 consecutive resections. Ann Surg 258(1): 129-140, 2013.

29 Friedl A, Stoesz SP, Buckley P and Gould MN: Neutrophil gelatinase-associated lipocalin in normal and neoplastic human tissues. Cell type-specific pattern of expression. Histochem J 31(7): 433-441, 1999.

30 Borkham-Kamphorst E, Drews F and Weiskirchen R: Induction of lipocalin-2 expression in acute and chronic experimental liver injury moderated by pro-inflammatory cytokines interleukin1beta through nuclear factor-kappaB activation. Liver Int 31(5): 656-665, 2011.

31 Wang W, Lin J, Guha S, Tong Z, Cameron AG, Zhang F, Qiu X, Zou C, Gao X, Mawad ME and Ke S: Target-specific agents imaging ectopic and orthotopic human pancreatic cancer xenografts. Pancreas 40(5): 689-694, 2011.

32 Chen W, Ayala-Orozco C, Biswal NC, Perez-Torres C, Bartels M, Bardhan R, Stinnet G, Liu XD, Ji B, Deorukhkar A, Brown LV, Guha S, Pautler RG, Krishnan S, Halas NJ and Joshi A: Targeting pancreatic cancer with magneto-fluorescent theranostic gold nanoshells. Nanomedicine 9(8): 1209-1222, 2014.

33 Glaser SS, Gaudio E and Alpini G: Vascular factors, angiogenesis and biliary tract disease. Curr Opin Gastroenterol 26(3): 246-250, 2010.

34 Weis SM and Cheresh DA: Tumor angiogenesis: Molecular pathways and therapeutic targets. Nat Med 17(11): 1359-1370, 2011. 